Published: Journal of Derivatives, Vol 5, No. 3, Spring 1998, pp 9-19

\title{
VALUE AT RISK WHEN DAILY CHANGES IN MARKET VARIABLES ARE NOT NORMALLY DISTRIBUTED
}

\author{
John Hull and Alan White*
}

First Draft: March, 1997

This Version: November, 1997

This paper proposes a new model for calculating VaR where the user is free to choose any probability distributions for daily changes in the market variables and parameters of the probability distributions are subject to updating schemes such as GARCH.

Transformations of the probability distributions are assumed to be multivariate normal. The model is appealing in that the calculation of VaR is relatively straightforward and can make use of the RiskMetrics or a similar database. We test a version of the model using nine years of daily data on 12 different exchange rates. When the first half of the data is used to estimate the model's parameters we find that it provides a good prediction of the distribution of daily changes in the second half of the data.

* Faculty of Management, University of Toronto, 105 St. George Street, Toronto, Ontario, Canada M5S 3E6. We are grateful to Tom McCurdy for comments and helpful suggestions. An earlier version of this paper was entitled "Taking account of the kurtosis in market variables when calculating value at risk" 


\title{
VALUE AT RISK WHEN DAILY CHANGES IN MARKET VARIABLES ARE NOT NORMALLY DISTRIBUTED
}

\author{
John Hull and Alan White
}

In the last few years Value at Risk (VaR) has become a very popular risk management tool in many different types of organizations. There are a number of reasons for this. One is J.P. Morgan's decision in 1994 to make their RiskMetrics database freely available to all market participants. Another is the climate created by derivatives disasters such as Procter and Gamble, Kidder Peabody, Orange County, and Barings. A third reason is the decision by central bank regulators to use $\mathrm{VaR}$ in calculating a bank's required capital. ${ }^{1}$

A VaR calculation is aimed at making a statement of the following form: "We are $X \%$ certain that we will not lose more than $V$ dollars in the next $N$ days." The variable $V$ is the VaR. It is a function of two parameters: $N$, the time horizon and $X$, the confidence level. In defining a bank's required capital, regulators use $N=10$ and $X=99$. The required capital is therefore based on the losses over a ten-day period that are expected to happen $1 \%$ of the time.

Models for calculating VaR are reviewed by Duffie and Pan (1997). The model most commonly used assumes that the probability distribution of the daily changes in each market variable is normal. However this assumption is far from perfect. The daily changes in many variables, particularly exchange rates, exhibit significant amounts of positive kurtosis. This means that the probability distributions of daily changes in these variables have "fat tails" so that extreme outcomes happen much more frequently than would be predicted by the normal distribution assumption. Duffie and Pan (1997) identify jumps and stochastic volatility as possible causes of kurtosis. They point out that under a jumpdiffusion model, kurtosis is a declining function of the time horizon whereas, under a stochastic volatility model, it is an increasing function of the time horizon (at least for the time horizons normally considered in VaR calculations).

In this article we show how the normal distribution assumption can be relaxed. We develop a model where the user is free to choose any probability distribution for the daily changes in each market variable and the parameters of the distribution are subject to updating schemes such as GARCH. A key aspect of the model is the way in which correlations are handled. We transform the daily changes in each market variable into a new variable that is normally distributed. We assume that the new variables are multivariate normal. We illustrate the approach using nine years of daily data on twelve different exchange rates.

Section I describes the usual VaR assumptions and reviews the main approaches to calculating VaR. Section II presents data on the kurtosis of major exchange rates. Section III describes the model. Section IV applies the model to exchange rate data. Section V

\footnotetext{
${ }^{1}$ See Jackson et al (1997) for a discussion of this.
} 
explains how the model can be used in conjunction with the RiskMetrics or similar database. Conclusions are in Section VI.

\section{Approaches to Calculating Value at Risk}

There are a number of alternative ways to calculate $\mathrm{VaR}$ for a portfolio. A popular approach that uses the RiskMetrics or similar database is to assume a model where the changes in the values of the market variables (equity prices, zero-coupon bond prices, exchange rates, commodity prices, etc.) have a multivariate normal distribution. The mean change in the value of each variable is assumed to be zero.

If the instruments in the portfolio are linearly dependent on $n$ market variables, VaR can be calculated analytically when this model is used. The dollar change in the portfolio value in one day, $\Delta P$, has the form:

$$
\Delta P=\sum_{i=1}^{n} a_{i} \Delta x_{i}
$$

where $\Delta x_{i}$ is the proportional change in the value of the $i$ th market variable during the day and the $a_{\mathrm{i}}$ are constants $(1 \leq i \leq n)$. The probability distribution of the portfolio value at the end of one day is normal with mean zero and standard deviation $\sigma_{P}$ where

$$
\sigma_{P}^{2}=\sum_{i=1}^{n} \sum_{j=1}^{n} \rho_{i j} a_{i} a_{j} \sigma_{i} \sigma_{j}
$$

The variable $\sigma_{i}$ is the daily volatility of the $i$ th market variable and $\rho_{i j}$ is the correlation between $\Delta x_{i}$ and $\Delta x_{j}$. The $\mathrm{VaR}$ for any given confidence level and any time horizon can easily be calculated from $\sigma_{P}$. For example, the VaR with a confidence level of $99 \%$ and a horizon of $N$ days is $2.33 \sigma_{P} \sqrt{N}$.

When the portfolio includes instruments that are not linearly dependent on the market variables, there are no exact approaches to calculating $\mathrm{VaR}$. One possibility is to use Monte Carlo simulation. Unfortunately this can be quite time consuming since the complete portfolio must be revalued on each simulation trial. An alternative is to approximate the relationship between $\Delta P$ and the $\Delta x_{i}$ 's using the first two terms in a Taylor series expansion so that

$$
\Delta P=\sum_{i=1}^{n} \frac{\partial P}{\partial x_{i}} \Delta x_{i}+0.5 \sum_{i=1}^{n} \sum_{j=1}^{n} \frac{\partial^{2} P}{\partial x_{i} \partial x_{j}} \Delta x_{i} \Delta x_{j}
$$

This expression can be used to calculate the moments of $\Delta P$ analytically. ${ }^{2}$ Alternatively a Monte Carlo simulation can be used with $\Delta P$ being calculated directly from equation (1).

\footnotetext{
${ }^{2}$ The Cornish-Fisher expansion provides an approximate way of converting the moments of $\Delta P$ to the appropriate fractile of the distribution of $\Delta P$. For a description see Johnson and Kotz (1972).
} 
The latter is referred to as the partial simulation approach. It is much less time consuming that a full simulation since it avoids the need for the portfolio to be revalued on each simulation trial.

Recently Jamshidian and Zhu (1997) have suggested an interesting alternative way of speeding up Monte Carlo simulation. This is known as scenario simulation. It involves defining an $M$-point discrete approximation to the probability distribution of each market variable. On each simulation trial, samples for the changes in each market variable are taken from the full multivariate distribution of the market variables in the usual way. Each sample is then replaced by the closest value in the corresponding discrete distribution before valuing the portfolio. The advantage of the approach is that it significantly reduces the number of times individual instruments in a portfolio (particularly those dependent on only one market variable) have to be valued.

Some analysts prefer to use historical data rather than a model to define the statistical behavior of market variables. This involves creating a database consisting of the daily movements in all market variables over a period of time. The first simulation trial assumes that the percentage changes in each market variable are the same as those on the first day covered by the database; the second simulation trial assumes that they are the same as those on the second day; and so on. The change in the portfolio value is calculated for each simulation trial and the $\mathrm{VaR}$ is calculated as the appropriate fractile of the probability distribution of these portfolio changes. The change in the portfolio value can be obtained either by revaluing the portfolio or by using equation (1).

The historical data approach has the advantage that it accurately reflects the historical multivariate probability distribution of the market variables. Its disadvantage is that the number of simulation trials is limited to the number of days of data that are available. Also, sensitivity analyses are difficult and variables for which there are no market data cannot easily be included in the analysis. The purpose of this paper is to extend the modelbuilding approach so that the historical behavior of market variables is represented more accurately.

\section{Non-Normality in Market Variables}

Although the RiskMetrics VaR calculation approach assumes multivariate normality, the changes in many market variables, particularly exchange rates, exhibit positive kurtosis. This means that extreme movements in the variables are more likely than a normal distribution would predict. Exhibit 1 compares a normal distribution with a distribution exhibiting positive kurtosis. ${ }^{3}$ Both distributions have the same mean and variance. However, the positive-kurtosis distribution is more peaked and has fatter tails. It is interesting to note what happens when we move from a normal distribution to a distribution with positive kurtosis. Probability mass is added to the central part of the

\footnotetext{
${ }^{3}$ The positive kurtosis distribution in Figure 1 was constructed as an equally weighted mixture of two normal distributions.
} 
distribution and added to the tails of the distribution. At the same time probability mass is taken from regions of the probability distribution that are intermediate between the tails and the center. The effect of kurtosis is therefore to increase the probability of very large moves and very small moves in the value of the variable while decreasing the probability of moderate moves.

To illustrate the problem of non-normality in market variables we will examine the behavior of exchange rates. The data we will use in this paper consists of daily exchange rates for 12 major currencies between January 4, 1988 and August 15, 1997. The total number of trading days covered by the data is 2,425. The currencies are the Australian dollar (AUD), Belgian franc (BEF), Swiss franc (CHF), German deutschemark (DEM), Danish krone (DKK), Spanish peseta (ESP), French franc (FRF), British pound (GBP), Italian lire (ITL), Japanese yen (JPY), Dutch guilder (NGL), and Swedish krone (SEK).

Define $e_{i}$ as the proportional change in an exchange rate between day $i$ and day $i+1$. The table in Exhibit 2 shows the frequency with which $e_{i}$ exceeded 1, 2, 3, 4, 5, and 6 standard deviations for each of the currencies. (For the purposes of calculating this table, $e_{i}$ was assumed to have zero mean and constant variance.) The table illustrates that all currencies exhibit significant excess kurtosis. The hypothesis that the $e_{i}$ are normal can be rejected with a very high degree of confidence. The probability of a one standard deviation move is $25.04 \%$ on average. This is considerably less than the $31.73 \%$ predicted by the normal distribution and indicates that exchange rates have a more peaked distribution than the normal distribution. The probability of a three standard deviation move is $1.34 \%$ on average. This compares with $0.27 \%$ for the normal distribution and is consistent with exchange rates having fatter tails than the normal distribution. Exhibit 2 also shows the usual excess kurtosis measure for each currency. ${ }^{4}$ For a normal distribution this measure is zero.

The RiskMetrics database uses an exponentially weighted moving average (EWMA) for the daily variance so that

$$
\sigma_{i}^{2}=\lambda \sigma_{i-1}^{2}+(1-\lambda) e_{i-1}^{2}
$$

where $\sigma_{i}^{2}$ is the variance calculated on day $i$ and $\lambda$ is set equal to $0.94 .^{5}$ Exhibit 3 shows the frequency with which $e_{i}$ exceeded $1,2,3,4,5$, and 6 standard deviations when the standard deviation is calculated using equation (2). The table shows that the stochastic volatility model in equation (2) does lead to a significant reduction in the excess kurtosis measure. However, the discrepancies between the observed frequencies and those that would be expected if the $e_{i}$ were normal are still large. The hypothesis that the $e_{i}$ are normal can still be rejected with a very high degree of confidence.

\footnotetext{
${ }^{4}$ The excess kurtosis of a distribution is defined as

$$
\frac{m_{4}}{v^{2}}-3
$$

where $m_{4}$ is its fourth central moment and $v$ is its variance.

${ }^{5}$ This is a particular case of a $\operatorname{GARCH}(1,1)$ process.
} 


\section{Modeling Non-Normality and the Calculation of VaR}

The traditional approach to modeling non-normality in observed outcomes in a time series has been to assume that, although the unconditional returns are not normal, suitably conditioned returns are normal. There have been three popular models of this type: GARCH models, mixed jump-diffusion models, and Markov switching models. In the GARCH or stochastic volatility models, the returns are normal conditional on knowing the current variance. In the mixed jump-diffusion models the returns are normal conditional on there being no jump. In the Markov switching models the returns are normal conditional on knowing the current state. The models have the advantage that they allow one to take advantage of the simple properties of multivariate normal distributions.

In this paper we are proposing a fourth alternative for handling non-normality. Our proposal is that some functional transformation of the observed returns is normal. This is similar to the other three models in that, suitably conditioned, the data is assumed to be normal. The proposal is not entirely new. For example, it has traditionally been assumed that changes in asset prices are drawn from a lognormal distribution so that changes in the logarithm of the asset price are normally distributed.

Suppose that there are a total of $m$ market variables. Define $e_{i j}$ as the proportional change in variable $j$ on day $i(1 \leq j \leq m)$ and $G_{i j}$ is the cumulative probability distribution assumed for $e_{i j}$. In general, $G_{i j}$ is dependent on a number of parameters, some independent of $i$ and some subject to updating schemes similar to GARCH. We transform $e_{i j}$ into a new variable $f_{i j}$ using the transformation

$$
f_{i j}=N^{-1}\left[G_{i j}\left(e_{i j}\right)\right]
$$

where $N$ is the cumulative normal distribution function. ${ }^{6}$ The expression inside the square brackets in equation (3) is the cumulative probability that the daily change in variable $j$ on day $i$ is less than $e_{i j}$. Denote this by $z$ so that $e_{i j}$ is the $z$-th fractile of the distribution being assumed for daily changes. The variable $f_{i j}$ is the same fractile of the standard normal distribution. In moving from $e_{i j}$ to $f_{i j}$ we are mapping observations from the assumed distribution of daily changes into a standard normal distribution on a "fractile-to-fractile" basis.

This approach can be used in conjunction with either the historical data approach or the model approach to VaR calculations. The model approach to VaR calculations assumes a particular form for the distribution of outcomes (for example, a displaced chi-square distribution, a t distribution, or a mixture-of-normals distribution.) and estimates the parameters of the distribution for each market variable. In this case, the $G$ function is calculated from the assumed distribution. The historical data approach bases the distribution of future changes on the distribution of past changes. In this case the $G$ function is the cumulative histogram of historic changes. We assume that the $f_{i j}(1 \leq j \leq m)$ have a multivariate normal distribution. $\mathrm{VaR}$ can be calculated using either Monte Carlo simulation or a Taylor Series expansion.

${ }^{6}$ A similar idea is in Hull (1977) and has been used by Duan (1997) 
When Monte Carlo simulation is used, the multivariate normally distributed variables, $f_{i j}$, are mapped into actual outcomes, $e_{i j}$, using

$$
e_{i j}=G_{i j}^{-1}\left[N\left(f_{i j}\right)\right]
$$

On each simulation trial, we sample an $f_{i j}$ and from equation (4) find the corresponding $e_{i j}{ }^{7}$ The $e_{i j}$ are then used to calculate the change in the value of the portfolio in the usual way, using either equation (1) or a full portfolio revaluation.

This model is ideally suited to be used in conjunction with the scenario simulation approach of Jamshidian and Zhu (1997) described earlier. We first replace the assumed continuous probability distribution for each $e$-variable by a discrete distribution in a way similar to that suggested by Jamshidian and Zhu. We then carry out a preliminary analysis to determine from equation (3) the range of values of $f_{i j}$ that correspond to each discrete value of $e_{i j}$. As the simulation proceeds, we move directly from samples of the $f_{i j}$ that come from a continuous multivariate normal to the corresponding samples of the $e_{i j}$ that come from the discrete approximations to the assumed distributions.

To use the Taylor Series expansion we assume a quadratic relationship between the change in the portfolio value and the $e_{i j}$. To calculate the first few moments of the change in the portfolio value (so that the Cornish-Fisher expansion can be used) we require terms of the form $E\left(e_{i j}\right), E\left(e_{i j} e_{i k}\right), E\left(e_{i j} e_{i k}{ }^{2}\right), E\left(e_{i j}{ }^{2} e_{i k}{ }^{2}\right)$, etc. These can be calculated, tabulated and stored in advance of VaR calculations by carrying out a single Monte Carlo simulation of the $e_{i j}$ 's. If this Monte Carlo simulation has to be carried out every day, the Taylor Series expansion approach is clearly inferior to the use of Monte Carlo simulation approach in conjunction with a quadratic approximation. However, if only one parameter in $G_{i j}$ is updated from day to day, and this is a variance parameter that effectively "scales" the $e_{i j}$, then the Monte Carlo simulation has to be carried out only once (or at least only when the $G_{i j}$ functions are changed). This is the situation in the example given below and is likely to be the case in most applications of our approach. An alternative approach is to assume that the correlation between $e_{i j}{ }^{m}$ and $e_{i k}{ }^{n}$ is the same as it would be if the $e$ 's were normally distributed and calculate the required expectations from these correlations and the moments of the assumed probability distributions of the $e$ 's.

\section{A Particular Example of the Model}

In this section we illustrate how the $G$ functions can be chosen. ${ }^{8}$ The model parameters are estimated from the foreign exchange data described earlier and the quality of approximation is then tested by comparing the model fit for a hold-out sample of data. We use a mixtures-of-normals distribution to represent the $G$ functions. ${ }^{9}$ We consider two

\footnotetext{
${ }^{7}$ This may involve an iterative search.

${ }^{8}$ Our objective is to show that it is not difficult to find a reasonable representation of the actual distribution of returns for foreign exchange data. We are not attempting to find the "true" form of $G$.

${ }^{9}$ Other researchers who have used mixtures of normals in VaR calculations are Zangari (1996) and Venkataraman (1997). Zangari assumes probability distributions for each of the parameters describing the
} 
models. In the first model the distribution of daily changes is stationary. In the second model, the variance of the distribution of daily changes can change, but parameters describing the kurtosis of the distribution are constant. The parameter estimation is done by fitting the fractiles of the distribution. We emphasize that the functional form for the $G$ functions and the estimation procedure are illustrations of the general approach that can be followed. Many other different assumptions and estimation procedures can be used. However, it is encouraging that the results we will present for the second model are quite good.

Define $\sigma_{i}{ }^{2}$ as the variance of the proportional daily change $e_{i}$ in a variable on day $i$. We assume that the probability density of daily changes is ${ }^{10}$

$$
g_{i}\left(e_{i}\right)=\frac{p}{\sqrt{2 \pi} u \sigma_{i}} \exp \left(-\frac{e_{i}^{2}}{2 u^{2} \sigma_{i}^{2}}\right)+\frac{1-p}{\sqrt{2 \pi} u \sigma_{i}} \exp \left(-\frac{e_{i}^{2}}{2 v^{2} \sigma_{i}^{2}}\right)
$$

so that

$$
G_{i}\left(e_{i}\right)=p N\left(\frac{e_{i}}{u \sigma_{i}}\right)+(1-p) N\left(\frac{e_{i}}{v \sigma_{i}}\right)
$$

The probability density function is a weighted average of two zero-mean normal distributions. The first distribution has weight $p$ and standard deviation $u \sigma_{i}$. The second distribution has weight 1- $p$ and standard deviation $v \sigma_{i}$. Note that we are not assuming a Markov switching model where on any given day either one normal distribution or the other applies. Equation (5) defines a family of distributions in terms of four parameters: $p$, $u, v$, and $\sigma_{i}$.

The variance of the distribution in (5) is

$$
p u^{2} \sigma_{i}{ }^{2}+(1-p) v^{2} \sigma_{i}{ }^{2}
$$

Since this must equal $\sigma_{i}^{2}$, the parameters underlying the distribution must satisfy

$$
p u^{2}+(1-p) v^{2}=1
$$

In fitting the model to the data we consider two cases. In the first case, the variance of daily changes in a market variable is assumed to be constant and equal to $\sigma^{2}$ so that $\sigma_{i}=$ $\sigma$. (This is the assumption in Exhibit 2.) In the second case the variance is assumed to be given by the EWMA model in equation (2) with $\lambda=0.94$. Other GARCH updating schemes for $\sigma_{i}$ could also be considered.

To test the models we chose values of $p, u$, and $v$ that are consistent with equation (6) and provide a best fit to the distribution of $e_{i}$ over the period covered by the first half of the

mixture of normals and uses a Bayesian updating scheme. Venkataraman uses a quasi-Bayesian maximum likelihood estimation procedure. Our model is non-Bayesian and conceptually simpler than that of either Zangari or Venkataraman. Also it has a quite different approach to handling correlations.

${ }^{10}$ For ease of notation we omit the $j$ subscript in this part of the paper. 
data (January 4, 1988 to October 19, 1992). A natural approach here would appear to be to maximize the log-likelihood function:

$$
\sum_{i} \log \left[\frac{p}{u \sigma_{i}} \exp \left(-\frac{e_{i}^{2}}{2 u^{2} \sigma_{i}^{2}}\right)+\frac{1-p}{v \sigma_{i}} \exp \left(-\frac{e_{i}^{2}}{2 v^{2} \sigma_{i}^{2}}\right)\right]
$$

One problem with this, pointed out by Hamilton (1991), is that attempts to maximize the function can lead to instability, local solutions, and non-convergence problems. ${ }^{11}$ Another problem is that the best fit values of $p, u$, and $v$ are greatly influenced by extreme data items. $^{12}$

To overcome these problems, we decided to estimate the parameters by fitting the fractiles of the distribution. The data on exchange rate changes was divided into four categories: less than one standard deviation $\left(\left|e_{i}\right| \leq \sigma_{i}\right)$; one to two standard deviations $\left(\sigma_{i}<\left|e_{i}\right| \leq 2 \sigma_{i}\right)$; two to three standard deviations $\left(2 \sigma_{i}<\left|e_{i}\right| \leq 3 \sigma_{i}\right)$; and, greater than three standard deviations $\left(3 \sigma_{i}<\left|e_{i}\right|\right)$. We compared the number of data items observed in each category with the number that would be predicted for particular values of $p, u$, and $v$ and selected the values of these parameters that maximize the log-likelihood function ${ }^{13}$

$$
\sum_{k=1}^{4} \alpha_{k} \log \left(\beta_{k}\right)
$$

where $\alpha_{k}$ is the actual proportion of observations in the $k$ th category and $\beta_{k}$ is the predicted proportion. This procedure was carried out for each of the 12 currencies as well as for the pooled results from all exchange rates. Parameter estimates were made for the constant variance case and the EWMA case. The best fit parameters for the EWMA case values are shown in Exhibit 4.

The best-fit estimates of $p, u$, and $v$ were then assumed to apply to the second half of the data sample and a chi-square statistic was calculated to determine the goodness of fit. For the purposes of calculating the chi-square statistic the data was categorized as described

\footnotetext{
${ }^{11}$ Consider for example the situation where one of the $e_{\mathrm{i}}$ is zero. As $u$ tends to zero the two exponential functions are both 1 , while $p /\left(u \sigma_{i}\right)$ tends to infinity so that the log-likelihood function also tends to infinity.

${ }^{12}$ It might seem strange that we are concerned about the adverse effect of extreme data items on our parameter estimates when the purpose of the model is to capture the probability of extreme outcomes more accurately. Our argument is as follows. When calculating VaR, we are not interested in modeling the really extreme (e.g. $0.1 \%$ tails) of the distribution of a market variable accurately at the expense of a decrease in the accuracy with which the $1 \%$ and $5 \%$ tails are modeled. This is because the $0.1 \%$ tail is not known accurately from historical data and does not influence a VaR calculation for the confidence levels usually chosen.

${ }^{13}$ The probability that, out of a total of $N$ observations, $n_{i}$ are in category $i(1 \leq i \leq 4)$ is proportional to
}

$$
\beta_{1}^{n_{i}} \beta_{2}^{n_{2}} \beta_{3}^{n_{3}} \beta_{4}^{n_{4}}
$$

Taking the logarithm of this function and dividing by $N$ we obtain the log-likelihood function. 
above. ${ }^{14}$ We carried out a separate analysis for each of the 12 currencies. For the parameters estimated from the pooled results from all exchange rates, the chi-square statistic was calculated by applying the pooled parameter estimates to each currency separately. These results for the EWMA case also appear in Exhibit 4. The results for the constant variance case are poor due to substantial changes in the volatilities of many of the currencies between the first period and the second period. ${ }^{15}$

In testing the validity of the model and the estimated parameters for a single currency, the model can be rejected with $95 \%$ confidence when the chi-square statistic is greater than 7.8. In the case in which we attempt to fit the distributions of all currencies simultaneously, the model can be rejected with $95 \%$ confidence when the chi-square statistic is greater than 51. Exhibit 4 shows that for the exponentially weighted moving average model the single currency model can be rejected for only four of the currencies. When the same $p, u$, and $v$ is used for all currencies, the value of the chi-square statistic is 48.24 indicating that the model cannot be rejected with $95 \%$ confidence. The sum of the chi-squares for the individual currency models is 72.73 , indicating that we can reject at the 95\% confidence level the hypothesis that the EWMA model is correct with a different $p$, $u$, and $v$ for each currency.

Our analysis therefore provides support for using the EWMA model with same $p, u$, and $v$ parameters for all currencies. The fit to the first half of the data is of course slightly worse when the $p, u$, and $v$ parameters are constrained to be the same for all currencies. ${ }^{16}$

However, the fit to the second half of the data is much better. This is because the best fit parameter values for an individual currency are not particularly stable whereas the best fit parameters for the all-currency model are quite stable. For the exponentially weighted moving average model the latter change from $p=0.62, u=0.70$, and $v=1.36$ for the first half of the data to $p=0.66, u=0.73$, and $v=1.38$ for the second half.

Exhibit 5 compares the actual distributions of $e_{i}$ for the second half of the data assuming the EWMA model with the distributions predicted by the normal distribution and by a model based on a single set of $p, u$ and $v$ parameters. The table illustrates that the mixture

14 The chi-square statistic is

$$
\sum_{i=1}^{4} \frac{\left(A_{i}-E_{i}\right)^{2}}{E_{i}}
$$

where $A_{i}$ is the actual number of observations in category $i$ in the second half of the data and $E_{i}$ is the expected number of observations based on the distribution estimated from the first half of the data.

${ }^{15}$ It is interesting to note that, if we base the calculation of the chi-square statistic on the volatility estimate of each currency during the second period instead of the first, the model works very well. Among the 12 currencies the constant variance version of the model can be rejected only for the Swedish krone. When a single model is used for all currencies the chi-square statistic is 29.55 , well under the $95 \%$ confidence level of 51. This suggests that, although the variance of a currency's return is non-stationary, the $p, u$, and $v$ parameters describing the kurtosis are fairly stationary.

${ }^{16}$ The maximum value of the total log-likelihood function (all currencies) decreases from -9.46 to -9.47 when we constrain the parameters to be the same for all currencies in the case of the exponentially weighted moving average model. 
of normals is a big improvement over the pure normal as a representation of daily exchange rate changes.

\section{Using the RiskMetrics or Similar Database}

Exhibit 6 shows estimates for the correlations between the $e$ 's and the correlations between the $f$ 's for the second half of the data in our example when the EWMA model is used to update variance estimates. The correlations are very similar. ${ }^{17}$ This suggests that, rather than estimating the correlation between the $f$ 's directly, we can reasonably assume that the correlation matrix for the $f$ 's is the same as that for the $e$ 's.

With this small approximation all calculations can be carried out using the Riskmetrics or a similar database once the $G$ functions have been determined. In general $G_{i j}$ depends on the estimate of the standard deviation of $e_{i j}$ made on day $i$. Our example used an EWMA model with $\lambda=0.94$ for this but other models can be used. The covariance matrix for the $f$ 's is known since, by construction the variance of each $f$ is 1.0 and by assumption the correlations between the $f$ 's are the same as those between the $e$ 's.

\section{Conclusions}

In this article we have shown how the usual multivariate-normal assumption in the calculation of VaR can be replaced by a "transform-to-multivariate-normal" assumption. The new model has the advantage that it enables the third, fourth and higher moments of the returns on market variables to be reflected in VaR calculations. We have illustrated the model using nine years of daily exchange-rate data for 12 currencies and find that it capable of accurately reflecting the probabilities of 1,2, and 3 standard deviation moves in the exchange rates. The model can be applied to calculating VaR relatively easily using the the RiskMetrics or a similar database. Either a Monte Carlo simulation or a Taylor Series expansion approach can be used.

\footnotetext{
${ }^{17}$ Note that the instantaneous correlations between the $e$ 's are identical to those between the $f$ 's.
} 


\section{References}

Duan , J-C, "Conditionally fat-tailed distributions and the volatility smile in options," Working Paper, Hong Kong University of Science and Technology, 1997.

Duffie, D. and J. Pan, “An overview of value at risk" Journal of Derivatives, Vol. 4, No. 3 (Spring 1997), pp. 7-49.

Hamilton, J. "A quasi-Bayesian approach to estimating parameters for mixtures of normal distributions" Journal of Business and Economic Statistics, Vol. 9, No. 1, 1991, pp. 2739.

Hull, J. C. "Dealing with dependence in risk simulations" Operational Research Quarterly, Vol 28, No. 1 ii, pp 201-203.

Jackson, P., D. J. Maude, and W. Perraudin "Bank capital and value at risk" Journal of Derivatives, Vol. 4 No. 3 (Spring 1997), pp 73-90.

Jamshidian, F. and Y. Zhu, "Scenario simulation model: theory and methodology" Finance and Stochastics, Vol. 1, 1997, pp. 43-67.

Johnson, S. L. and S. Kotz Distributions in statistics: Continuous univariate distributions 1. New York: John Wiley and Sons, 1972.

Venkataraman, S. "Value at risk for a mixture of normal distributions: the use of quasiBayesian estimation techniques" Economic Perspectives, Federal Reserve Bank of Chicago, March/April 1997, pp. 2-13

Zangari, P. “An improved methodology for measuring VaR” RiskMetrics Monitor, Reuters/JP Morgan, 1996. 


\section{EXHIBIT 1}

Comparison of Normal Distribution and Positive-Kurtosis Distribution. The Distributions have the Same Mean and Variance

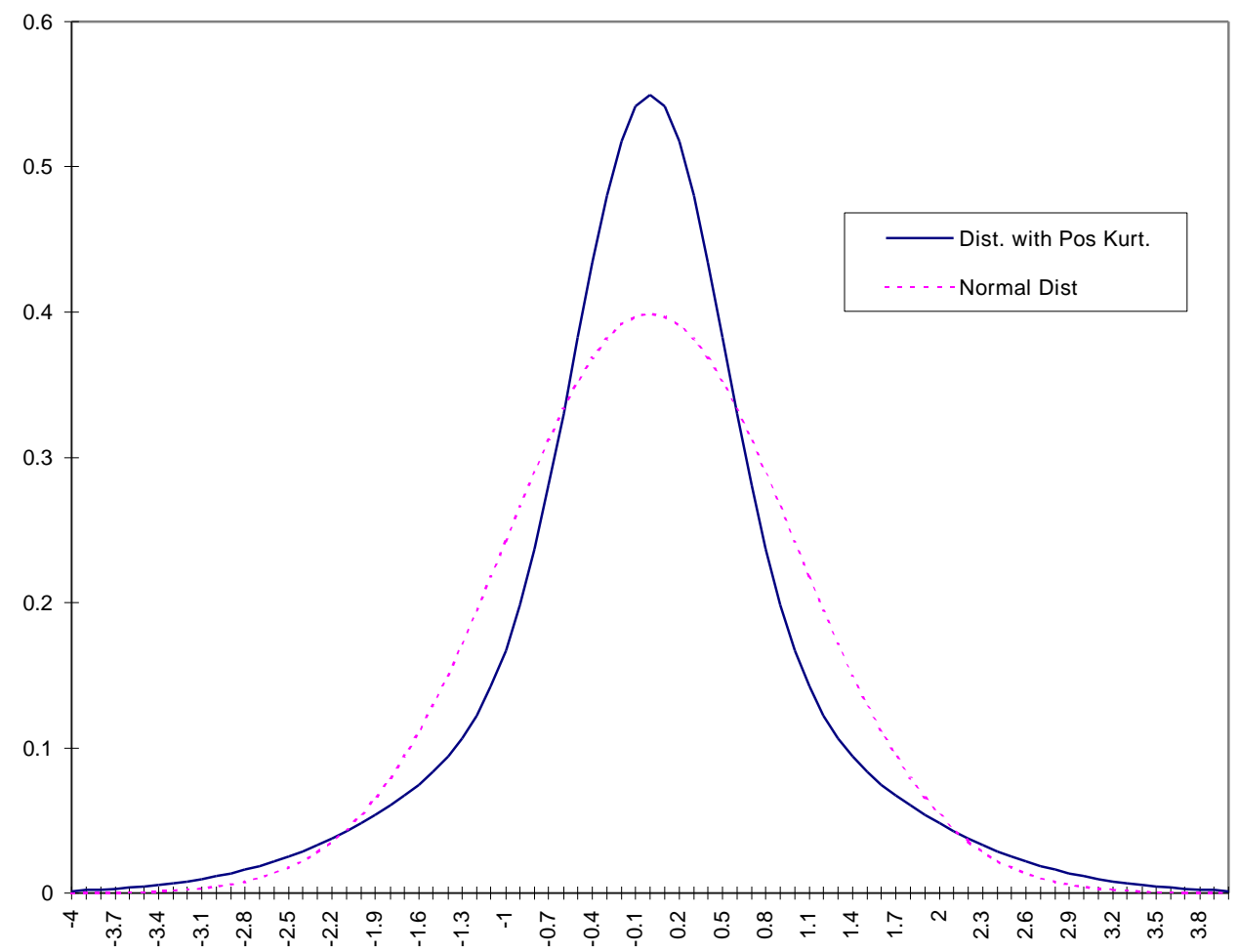


EXHIBIT 2

Exchange Rate Moves when Constant Variance Model is Used

\begin{tabular}{lrrrrrr}
\hline & \multicolumn{1}{l}{ AUD } & \multicolumn{1}{l}{ BEF } & \multicolumn{1}{l}{ CHF } & \multicolumn{1}{l}{ DEM } & \multicolumn{1}{l}{ DKK } & \multicolumn{1}{l}{ ESP } \\
\hline$>1$ stdev & $23.80 \%$ & $24.96 \%$ & $26.24 \%$ & $25.74 \%$ & $25.58 \%$ & $23.72 \%$ \\
$>2$ stdev & $5.49 \%$ & $4.95 \%$ & $5.45 \%$ & $5.65 \%$ & $5.40 \%$ & $4.70 \%$ \\
$>3$ stdev & $1.36 \%$ & $1.32 \%$ & $1.24 \%$ & $1.24 \%$ & $1.32 \%$ & $1.53 \%$ \\
$>4$ stdev & $0.41 \%$ & $0.25 \%$ & $0.17 \%$ & $0.25 \%$ & $0.12 \%$ & $0.37 \%$ \\
$>5$ stdev & $0.21 \%$ & $0.04 \%$ & $0.04 \%$ & $0.00 \%$ & $0.00 \%$ & $0.17 \%$ \\
$>6$ stdev & $0.08 \%$ & $0.04 \%$ & $0.00 \%$ & $0.00 \%$ & $0.00 \%$ & $0.08 \%$ \\
Kurtosis & 4.40 & 3.31 & 1.69 & 1.91 & 1.61 & 7.75 \\
\hline
\end{tabular}

\begin{tabular}{lrrrrrr}
\hline & \multicolumn{1}{l}{ FRF } & \multicolumn{1}{l}{ GBP } & \multicolumn{1}{l}{ ITL } & \multicolumn{1}{l}{ JPY } & \multicolumn{1}{l}{ NLG } & \multicolumn{1}{l}{ SEK } \\
\hline$>1$ stdev & $26.03 \%$ & $25.33 \%$ & $25.08 \%$ & $23.60 \%$ & $25.54 \%$ & $24.83 \%$ \\
$>2$ stdev & $5.53 \%$ & $5.45 \%$ & $4.83 \%$ & $5.40 \%$ & $5.24 \%$ & $5.20 \%$ \\
$>3$ stdev & $1.44 \%$ & $1.44 \%$ & $1.28 \%$ & $1.49 \%$ & $1.20 \%$ & $1.24 \%$ \\
$>4$ stdev & $0.12 \%$ & $0.37 \%$ & $0.29 \%$ & $0.62 \%$ & $0.25 \%$ & $0.25 \%$ \\
$>5$ stdev & $0.00 \%$ & $0.04 \%$ & $0.17 \%$ & $0.12 \%$ & $0.04 \%$ & $0.12 \%$ \\
$>6$ stdev & $0.00 \%$ & $0.00 \%$ & $0.08 \%$ & $0.00 \%$ & $0.00 \%$ & $0.04 \%$ \\
Kurtosis & 1.65 & 2.57 & 5.93 & 3.34 & 2.07 & 4.44 \\
\hline
\end{tabular}

\begin{tabular}{lrr}
\hline & Average & \multicolumn{1}{c}{ Normal } \\
\hline$>1$ stdev & $25.04 \%$ & $31.73 \%$ \\
$>2$ stdev & $5.27 \%$ & $4.55 \%$ \\
$>3$ stdev & $1.34 \%$ & $0.27 \%$ \\
$>4$ stdev & $0.29 \%$ & $0.01 \%$ \\
$>5$ stdev & $0.08 \%$ & $0.00 \%$ \\
$>6$ stdev & $0.03 \%$ & $0.00 \%$ \\
Kurtosis & 3.39 & 0.00 \\
\hline
\end{tabular}


EXHIBIT 3

Exchange Rate Moves When

Exponential Weighted Moving Average Model is Used

\begin{tabular}{lrrrrrr}
\hline & \multicolumn{1}{l}{ AUD } & \multicolumn{1}{l}{ BEF } & \multicolumn{1}{l}{ CHF } & \multicolumn{1}{l}{ DEM } & \multicolumn{1}{l}{ DKK } & \multicolumn{1}{l}{ ESP } \\
\hline$>1$ stdev & $26.98 \%$ & $28.42 \%$ & $28.80 \%$ & $28.55 \%$ & $29.17 \%$ & $29.00 \%$ \\
$>2$ stdev & $5.73 \%$ & $6.52 \%$ & $6.31 \%$ & $6.68 \%$ & $6.35 \%$ & $6.15 \%$ \\
$>3$ stdev & $1.53 \%$ & $1.40 \%$ & $1.03 \%$ & $1.44 \%$ & $1.28 \%$ & $1.32 \%$ \\
$>4$ stdev & $0.66 \%$ & $0.33 \%$ & $0.17 \%$ & $0.08 \%$ & $0.21 \%$ & $0.29 \%$ \\
$>5$ stdev & $0.21 \%$ & $0.08 \%$ & $0.08 \%$ & $0.04 \%$ & $0.04 \%$ & $0.12 \%$ \\
$>6$ stdev & $0.08 \%$ & $0.08 \%$ & $0.04 \%$ & $0.04 \%$ & $0.04 \%$ & $0.08 \%$ \\
Kurtosis & 3.59 & 3.23 & 2.09 & 2.03 & 1.97 & 2.77 \\
\hline
\end{tabular}

\begin{tabular}{lrrrrrr}
\hline & \multicolumn{1}{l}{ FRF } & \multicolumn{1}{l}{ GBP } & \multicolumn{1}{l}{ ITL } & \multicolumn{1}{l}{ JPY } & \multicolumn{1}{l}{ NLG } & \multicolumn{1}{l}{ SEK } \\
\hline$>1$ stdev & $29.50 \%$ & $27.81 \%$ & $28.96 \%$ & $26.49 \%$ & $29.37 \%$ & $29.29 \%$ \\
$>2$ stdev & $6.52 \%$ & $6.68 \%$ & $6.11 \%$ & $6.52 \%$ & $6.15 \%$ & $6.35 \%$ \\
$>3$ stdev & $1.53 \%$ & $1.44 \%$ & $1.69 \%$ & $1.24 \%$ & $1.11 \%$ & $1.24 \%$ \\
$>4$ stdev & $0.17 \%$ & $0.25 \%$ & $0.33 \%$ & $0.50 \%$ & $0.29 \%$ & $0.08 \%$ \\
$>5$ stdev & $0.04 \%$ & $0.08 \%$ & $0.08 \%$ & $0.17 \%$ & $0.08 \%$ & $0.04 \%$ \\
$>6$ stdev & $0.04 \%$ & $0.04 \%$ & $0.04 \%$ & $0.12 \%$ & $0.04 \%$ & $0.04 \%$ \\
Kurtosis & 2.12 & 2.35 & 2.15 & 3.47 & 2.15 & 1.47 \\
\hline
\end{tabular}

\begin{tabular}{lrr}
\hline & Average & \multicolumn{1}{c}{ Normal } \\
\hline$>1$ stdev & $28.53 \%$ & $31.73 \%$ \\
$>2$ stdev & $6.34 \%$ & $4.55 \%$ \\
$>3$ stdev & $1.36 \%$ & $0.27 \%$ \\
$>4$ stdev & $0.28 \%$ & $0.01 \%$ \\
$>5$ stdev & $0.09 \%$ & $0.00 \%$ \\
$>6$ stdev & $0.06 \%$ & $0.00 \%$ \\
Kurtosis & 2.45 & 0.00 \\
\hline
\end{tabular}




\section{EXHIBIT 4}

Best fit Values of $p, u$, and $v$ when the Exponentially Weighted Moving Average Mixture of Normals Model is Fitted to First Half of Data. The Chi-Square Statistic Measures the Goodness of Fit to the Second Half of the Data

\begin{tabular}{ccccccc}
\hline & AUD & BEF & CHF & DEM & DKK & ESP \\
\hline$p$ & 0.85 & 0.57 & 0.51 & 0.57 & 0.30 & 0.62 \\
$u$ & 0.78 & 0.69 & 0.69 & 0.66 & 0.14 & 0.68 \\
$1-p$ & 0.15 & 0.43 & 0.49 & 0.43 & 0.70 & 0.38 \\
$v$ & 1.77 & 1.30 & 1.24 & 1.32 & 1.19 & 1.37 \\
\hline Chi-Square & 5.81 & 6.14 & 1.90 & 1.86 & 9.24 & 9.92 \\
\hline
\end{tabular}

\begin{tabular}{cccccccc}
\hline & FRF & GBP & ITL & JPY & NLG & SEK & ALL \\
\hline$p$ & 0.49 & 0.35 & 0.68 & 0.75 & 0.79 & 0.48 & 0.62 \\
$u$ & 0.61 & 0.14 & 0.73 & 0.70 & 0.81 & 0.61 & 0.70 \\
$1-p$ & 0.51 & 0.65 & 0.32 & 0.25 & 0.21 & 0.52 & 0.38 \\
$v$ & 1.27 & 1.24 & 1.41 & 1.58 & 1.52 & 1.26 & 1.36 \\
\hline Chi-Square & 8.77 & 9.34 & 4.27 & 5.34 & 5.64 & 4.50 & 48.24 \\
\hline
\end{tabular}




\section{EXHIBIT 5}

Probability Distributions For Second Half of Data Compared With Probability Distribution for the Mixture of Normals EWMA Model that Provides Best Overall Fit to First Half of Data

\begin{tabular}{rrrrrrr}
\hline & AUD & BEF & CHF & DEM & DKK & ESP \\
\hline 0 to 1 SD & $72.44 \%$ & $72.19 \%$ & $72.28 \%$ & $72.11 \%$ & $70.96 \%$ & $70.30 \%$ \\
1 to 2 SD & $21.37 \%$ & $21.29 \%$ & $21.53 \%$ & $21.78 \%$ & $23.10 \%$ & $24.26 \%$ \\
2 to 3 SD & $4.70 \%$ & $4.95 \%$ & $5.20 \%$ & $4.79 \%$ & $4.54 \%$ & $4.46 \%$ \\
>3 SD & $1.49 \%$ & $1.57 \%$ & $0.99 \%$ & $1.32 \%$ & $1.40 \%$ & $0.99 \%$ \\
\hline
\end{tabular}

\begin{tabular}{rrrrrrr}
\hline & FRF & GBP & ITL & JPY & NLG & SEK \\
\hline 0 to 1 SD & $69.97 \%$ & $72.77 \%$ & $70.71 \%$ & $73.18 \%$ & $71.45 \%$ & $70.79 \%$ \\
1 to 2 SD & $24.09 \%$ & $21.04 \%$ & $23.51 \%$ & $20.05 \%$ & $22.36 \%$ & $23.68 \%$ \\
2 to 3 SD & $4.70 \%$ & $4.54 \%$ & $4.37 \%$ & $5.28 \%$ & $5.28 \%$ & $4.62 \%$ \\
$>$ 3 SD & $1.24 \%$ & $1.65 \%$ & $1.40 \%$ & $1.49 \%$ & $0.91 \%$ & $0.91 \%$ \\
\hline
\end{tabular}

\begin{tabular}{rrr}
\hline & $\begin{array}{c}\text { Best Fit } \\
\text { Model }\end{array}$ & $\begin{array}{c}\text { Normal } \\
\text { Model }\end{array}$ \\
\hline 0 to 1 SD & $73.11 \%$ & $68.27 \%$ \\
1 to 2 SD & $21.31 \%$ & $27.18 \%$ \\
2 to 3 SD & $4.55 \%$ & $4.28 \%$ \\
$>3$ SD & $1.03 \%$ & $0.27 \%$ \\
\hline
\end{tabular}




\section{EXHIBIT 6}

\section{Correlations between Exchange Rate Movements and between Transformed Exchange Rate Movements (Second Half of Data EWMA Model)}

\section{Exchange Rate Movements}

\begin{tabular}{|c|c|c|c|c|c|c|c|c|c|c|c|c|}
\hline & AUD & BEF & CHF & DEM & DKK & ESP & FRF & GBP & ITL & JPY & NLG & SEK \\
\hline AUD & 1.000 & & & & & & & & & & & \\
\hline BEF & -0.046 & 1.000 & & & & & & & & & & \\
\hline CHF & -0.054 & 0.862 & 1.000 & & & & & & & & & \\
\hline DEM & -0.057 & 0.926 & 0.916 & 1.000 & & & & & & & & \\
\hline DKK & -0.057 & 0.886 & 0.839 & 0.900 & 1.000 & & & & & & & \\
\hline ESP & -0.049 & 0.821 & 0.762 & 0.831 & 0.816 & 1.000 & & & & & & \\
\hline FRF & -0.055 & 0.924 & 0.887 & 0.945 & 0.902 & 0.846 & 1.000 & & & & & \\
\hline GBP & 0.087 & 0.566 & 0.576 & 0.605 & 0.586 & 0.526 & 0.599 & 1.000 & & & & \\
\hline ITL & 0.049 & 0.601 & 0.594 & 0.627 & 0.621 & 0.651 & 0.668 & 0.466 & 1.000 & & & \\
\hline JPY & -0.116 & 0.513 & 0.531 & 0.536 & 0.495 & 0.448 & 0.520 & 0.294 & 0.333 & 1.000 & & \\
\hline NLG & -0.059 & 0.934 & 0.914 & 0.984 & 0.901 & 0.836 & 0.946 & 0.595 & 0.628 & 0.534 & 1.000 & \\
\hline SEK & -0.006 & 0.573 & 0.571 & 0.600 & 0.598 & 0.578 & 0.625 & 0.449 & 0.588 & 0.324 & 0.594 & 1.000 \\
\hline
\end{tabular}

Transformed Exchange Rate Movements

\begin{tabular}{|c|c|c|c|c|c|c|c|c|c|c|c|c|}
\hline & AUD & BEF & CHF & DEM & DKK & ESP & FRF & GBP & ITL & JPY & NLG & SEK \\
\hline AUD & 1.000 & & & & & & & & & & & \\
\hline BEF & -0.053 & 1.000 & & & & & & & & & & \\
\hline CHF & -0.061 & 0.866 & 1.000 & & & & & & & & & \\
\hline DEM & -0.068 & 0.932 & 0.915 & 1.000 & & & & & & & & \\
\hline DKK & -0.071 & 0.897 & 0.844 & 0.908 & 1.000 & & & & & & & \\
\hline ESP & -0.055 & 0.823 & 0.765 & 0.833 & 0.820 & 1.000 & & & & & & \\
\hline FRF & -0.065 & 0.925 & 0.884 & 0.943 & 0.907 & 0.847 & 1.000 & & & & & \\
\hline GBP & 0.084 & 0.580 & 0.587 & 0.614 & 0.595 & 0.533 & 0.608 & 1.000 & & & & \\
\hline ITL & 0.037 & 0.606 & 0.600 & 0.627 & 0.629 & 0.657 & 0.672 & 0.478 & 1.000 & & & \\
\hline JPY & -0.123 & 0.513 & 0.528 & 0.532 & 0.498 & 0.451 & 0.516 & 0.299 & 0.339 & 1.000 & & \\
\hline NLG & -0.071 & 0.938 & 0.913 & 0.983 & 0.908 & 0.838 & 0.944 & 0.605 & 0.628 & 0.530 & 1.000 & \\
\hline SEK & -0.017 & 0.581 & 0.575 & 0.601 & 0.607 & 0.582 & 0.630 & 0.450 & 0.596 & 0.327 & 0.596 & 1.000 \\
\hline
\end{tabular}

